\title{
Modeling and Analysis of Random Periodic Spectrum Sensing for Cognitive Radio Networks
}

\author{
Caili GUO, Zhiming ZENG, Chunyan FENG, Qi LIU \\ School of Information and Communication Engineering, \\ Beijing University of Posts and Telecommunications, Beijing, China \\ Email: guocaili@bupt.edu.cn \\ Received June 18, 2009; revised August 20, 2009; accepted August 21, 2009
}

\begin{abstract}
A random periodic spectrum sensing scheme is proposed for cognitive radio networks. The sensing period, the transmission time for primary users and cognitive radios are extended to general forms as random variables. A generalized Markov analytical model for sensing period optimization is presented, and the applications of the proposed analytical model by using examples involving primary user systems with both voice and data traffic are illustrated. The analysis and numerical results show that sensing period does affect the maximum rewards of the channel, and the analytical model is justified by its flexibility since it uses general forms of the sensing period, the transmission time for primary users and cognitive radios. Hence the model can be easily adapted for the analysis of many different applications.
\end{abstract}

Keywords: Cognitive Radio Networks, Random Periodic Spectrum Sensing, Generalized Markov Process, the Optimal Sensing Period

\section{Introduction}

Due to energy consumption and hardware implication of Cognitive Radios (CRs), it is undesirable and impractical to assume the spectrum sensing to be continuous. In a practical CR network, such as an IEEE 802.22 network [1], a periodic spectrum sensing scheme where the spectrum is sensed periodically to determining the presence/absence of Primary Users (PUs) is preferable. The sensing time and sensing period are two key sensing parameters for periodic sensing scheme. The former is a pre-defined amount of time used to achieve the desirable level of detection quality and is mainly depended on PHY-layer sensing methods, such as energy detection, matched filter and feature detection. And the latter defined as the interval between two successive detection processes has a significant impact on the sensing efficiency of CRs. In the case of the sensing period is relatively large, both some opportunities may go undiscovered and interference to PUs may occur, whereas blindly reducing the sensing period is not desirable either, as it increases the sensing overhead. Thus the design of any

The material in this paper is based on "Random Periodic Spectrum Sensing with Sensing Period Optimization for Cognitive Radio Networks", by Caili Guo, Zhiming Zeng, Chunyan Feng and Qi Liu which appeared in the proceedings of 11th IEEE International Conference on Communications Systems, ICCS 2008, Guangzhou, China, November 2008 . periodic sensing scheme involves balancing the tradeoffs among spectrum utilization, interference to PUs, and sensing overhead by selecting an appropriate sensing period. We usually consider a spectrum consist of several channels, and each channel can be a frequency band with certain bandwidth, a spreading code in a CDMA network or a set of tones in an OFDM system. Here we use the term channel broadly.

In CR networks, the control of quiet period, during which all CRs should suspend their transmissions so that any CR monitoring the channel may observe the presence/absence of PU signals without interference, can be synchronous or asynchronous [1-2]. Accordingly there are two kinds of periodic sensing schemes: One is synchronous sensing period and the other is asynchronous sensing period. Most of the existing works focused on the synchronous sensing period schemes [3-4]. As a simple solution for design and implementation, the synchronous sensing period scheme sets a pre-determined fixed sensing period for all channels. While it does not need the scheduling of quiet period for each channel among CRs, it shows less flexible. Recent researches [5-7] showed that the asynchronous sensing period scheme is more favorable, in which sensing period can be adjusted adaptively according to the channel-usage characteristics of each channel by the MAC-layer sensing protocol or through a dedicated control channel [8]. Kim [5] proposed an adaptation algorithm in which the 
optimal sensing period is uniquely determined for each channel to maximize the discovery of opportunities as well as minimize the delay in locating an idle channel. However, this approach clearly did not consider the impact of sensing period selection on interference to PUs. In [6], we extended [5] to a Flexible Sensing Period (FSP) mechanism that introduces the "period control factor" to control each channel's sensing period adaptively to tradeoff undiscovered opportunities and interference to PUs with sensing overhead effectively, but as far as each channel is concerned, the FSP also consider that sensing period is still fixed. In order to combat the fluctuation of sensing period induced by the varying of channel-usage characteristics, in [7] we described a Fuzzy Spectrum Sensing Period Optimization (FSSPO) algorithm where each channel's sensing period is adaptively adjusted in real time with fuzzy logic and parameters optimization.

Existing approaches of asynchronous sensing period in $[5,6]$ and [7] only considered how to adjust sensing period which is usually regarded as a constant once it is determined, and they all assumed that the sensing results are perfect. In this paper, the random periodic sensing scheme we proposed extends the sensing period, the time of transmission for primary users and cognitive radios to random variables and more practical situation where sensing error exists is considered. As the PUs have the highest priority, we also introduce a back-off mechanism where a random back-off time is generated whenever PUs release the channel and CRs have to delay for the back-off time before occupying the channel. Here we focus on how to model the proposed random periodic sensing scheme to a generalized Markov process and how to derive the optimal sensing period. To support the proposed analytical model for sensing period optimization, we also illustrate the applications of the analytical model by using examples involving PU systems with both voice and data traffic.

The rest of the paper is organized as follows. Section 2 introduces the random periodic spectrum sensing scheme. A generalized Markov analytical model for sensing period optimization is constructed in Section 3. Then in Section 4, how to obtain performance measures of channels is considered. Example applications of the proposed analytical model for real networks are illustrated in Section 5. Numerical examples are presented and discussed in Section 6. Finally, we conclude the paper and suggest future directions in Section 7.

\section{Random Periodic Spectrum Sensing Scheme}

In CR networks, a channel usually could be modeled as an ON-OFF source alternating between ON (busy) and OFF (idle) periods depending on PUs' channel-usage pattern. The sojourn time of a ON period is used for transmission of PUs themselves and that of a OFF period captures the time period in which the channel can be utilized by CRs' transmission without causing any harmful interference to PUs. The distribution of the sojourn time in the ON state can assumed to be general, and so is that in the OFF state. Thus the ON-OFF channel-usage stochastic process describing the behavior of the channel occupation can form an alternative renewal process. A renewal period models a time period in which the PUs and CRs occupy the channel once alternatively. Hence there are only busy and idle two possible states in a renewal period accordingly.

Considering that the sensing period of each channel is a random variable and sensing errors are possible present at any moment as well as a back-off mechanism is introduced, in this paper we further subdivide the channel in a renewal period into five kinds of states: normal busy, available idle, delay idle, false alarm and miss detection. Below we will describe each state in detail.

Normal busy and available idle are two kinds of normal available states. The former denotes that PUs are being served normally and the latter stands for the channel are being utilized for the transmission of CRs. When the channel is in normal available, it is sensed once every random time interval $T$, i.e. sensing period, to make sure whether it is in normal busy or available idle.

As soon as the service of PUs completes, a random back-off time interval $T_{0}$ is generated to prevent CRs from occupying the channel at once, within which the channel is in delay idle state. The back-off mechanism can help decrease both the connection cost generated by switching channel state frequently and the short-term interference probability induced by non-negligible delay for relinquishing bands by CRs.

Corresponding to the binary hypotheses test of spectrum sensing: $B_{0}$ (null hypothesis indicating that the sensed channel is available for CRs) vs. $B_{1}$ (alternative), there are two kinds of sensing errors: false alarm (the overlook of an available channel) due to mistaking $B_{0}$ for $B_{1}$ and miss detection (the mistake of identifying an unavailable channel as an opportunity) due to mistaking $B_{1}$ for $B_{0}$. When false alarm or miss detection is present, the channel will transfer to false alarm or miss detection state. The presence of sensing errors also has significant effects on the performance of sensing schemes.

Common to most of the periodic spectrum sensing schemes, the sensing period selection of random periodic spectrum sensing scheme has strong impact on the sensing efficiency. In order to analyze the optimal sensing period effectively, an analytical model for sensing period optimization is constructed in next section.

\section{Analytical Model}

Owing to the multiplicity of conditionality and correlation that exists among the various random variable in- 
volved in the random periodic sensing scheme, the analysis and performance evaluation, especially the determination of optimal sensing period is usually difficult. Therefore, a set of simplifying assumptions is to be made for analytical model to be tractable. We assume the following

- The sojourn time of normal busy and available idle are continuous random variables and drawn from general cumulative distribution functions (c.d.f.) represented by $F_{1}(t)$ and $F_{2}(t)$ respectively. Suppose the probability density functions (p.d.f.) for $\mathrm{F}_{\mathrm{i}}(\mathrm{t})(i=1,2)$ are $f_{\mathrm{i}}(t)$, means are $\omega_{i}^{-1}$, and $F_{i}(t)=\int_{0}^{t} f_{i}(x) d x=1-\exp \left[-\int_{0}^{t} \omega_{i}(x) d x\right]$, $\omega_{i}^{-1}=\int_{0}^{\infty} t d F_{i}(t)$.

- The sensing period $T$ is also a continuous random variable and follows an arbitrary distribution with c.d.f., p.d.f. and mean are $G_{1}(t), g_{1}(y)$ and $v_{1}^{-1}$, respectively, and $G_{1}(t)=\int_{0}^{t} g_{1}(y) d y=1-\exp \left[-\int_{0}^{t} v_{1}(y) d y\right] \quad E(T)=v_{1}^{-1}$ $=\int_{0}^{\infty} t d G_{1}(t)$.

- Delay idle state can transfer to normal busy or available idle. It is assumed that if a PU reappears during $T_{0}$ he can claim the channel and the delay idle will transfer to normal busy with a constant rate $\gamma$. Otherwise, if the back-off timer expires and no one claims the channel, delay idle transfers to available idle instead. In this case, $T_{0}$ follows an arbitrary distribution, and its c.d.f., p.d.f. and mean are $G_{2}(t), g_{2}(y)$ and $v_{2}^{-1}$, respectively, and

$$
\begin{gathered}
G_{2}(t)=\int_{0}^{t} g_{2}(y) d y=1-\exp \left[-\int_{0}^{t} v_{2}(y) d y\right] \\
E\left(T_{0}\right)=v_{2}{ }^{-1}=\int_{0}^{\infty} t d G_{2}(t)
\end{gathered}
$$

- We assume that $\alpha$ and $\beta$ denote false alarm and miss detection probabilities respectively, and the sojourn times of false alarm and miss detection are arbitrarily distributed with c.d.f.s $H_{i}(t), i=1,2$. Let $h_{i}(t)$ and $\varphi_{i}^{-1}$ be their p.d.f.s and means, and

$$
H_{i}(t)=\int_{0}^{t} h_{i}(z) d z=1-\exp \left[-\int_{0}^{t} \varphi_{i}(z) d z\right], \varphi_{i}^{-1}=\int_{0}^{\infty} t d H_{i}(t)
$$

When miss detection occurs the channel transfers to delay idle, whereas when false alarm occurs, in order to render mathematical tractability, we also assume that the channel skips normal occupy and transfers to delay idle too, for the normal occupy time included is small enough compared to total time of the long-run channel and can be neglected.

- We assume that the sensing time is small relative to distribution parameters $\omega_{1}, \omega_{2}, \gamma, E(T), E\left(T_{0}\right)$ and $\varphi_{i}{ }^{-1}(i=1,2)$, and can be negligible. It is also assumed that channel is in delay idle initially, and all random variables are mutually independent.

Consider the stochastic process $\{S(t), t \geq 0\}+$, where $S(t)$ denotes the state of the channel at time $t$, as following
- 0 stands for the channel is in delay idle, and the channel is occupied neither by PUs nor by CRs.

- $(i, k)$ means that the channel is in normal available states, where $i=0 / 1$ represents that the channel is in available idle/normal busy and $k$ stands for the times of the channel has been sensed, $\mathrm{k}=0,1,2, \cdots$.

- $(2, j)$ denotes that the sensing error is present, where $j=1 / 2$ means that the system is in false alarm $/$ miss detection.

It is easy to see that, $\{S(t), t \geqslant 0\}$ is a non-Markovian stochastic process. In order to make the process Markovian, we need to incorporate the missing information by adding "supplementary variables" to the state description. Hence at time t, let $X_{i}(t)(i=1 / 2)$ be the remaining normal busy/available idle time, $Y_{i}(t)(i=1 / 2)$ the remaining sensing/delay time, and $Z_{i}(t)(i=1 / 2)$ the remaining false alarm /miss detection time. Formally, the evolution of the stochastic process describing the dynamic behavior of the channel can be fully characterized by a generalized Markov process $\left\{S(t), X_{i}(t), Y_{i}(t), Z_{i}(t) \mid t \geq 0\right\}$, and the following state probabilities are defined

$$
\left\{\begin{array}{c}
P_{i k}(t, x, y) d x=P\left\{S(t)=(i, k), x<X_{i}(t) \leq x+d x,\right. \\
\left.\quad y<Y_{1}(t) \leq y+d y\right\}, i=0,1 ; k=0,1,2, \cdots \\
P_{0}(t, y) d y=P\left\{S(t)=0, y<Y_{2}(t) \leq y+d y\right\} \\
P_{2 j}(t, z) d z=P\left\{S(t)=(2, j), z<Z_{i}(t) \leq z+d z\right\}, j=1,2
\end{array}\right.
$$

Notations:

$$
\begin{gathered}
-\bar{F}_{i}(t)=1-F_{i}(t), *: f_{i}^{*}(s)=\int_{0}^{\infty} f_{i}(t) e^{-s t} d t, \bar{F}_{i}^{*}(s)=\left[1-f_{i}^{*}(s)\right] / s \\
f_{\omega 1}, f_{\omega 2}, g_{\gamma}: f_{\omega 1}=f^{*}\left(s+\omega_{1}\right), f_{\omega 2}=f^{*}\left(s+\omega_{2}\right), g_{\gamma}=g^{*}(s+\gamma) \\
\hat{f}_{\omega 1}, \hat{f}_{\omega 2}, \hat{g}_{\gamma}: \hat{f}_{\omega 1}=f^{*}\left(\omega_{1}\right), \hat{f}_{\omega 2}=f^{*}\left(\omega_{2}\right), \hat{g}_{\gamma}=g^{*}(\gamma) \\
\bar{\alpha}, \bar{\beta}: \bar{\alpha}=1-\alpha, \bar{\beta}=1-\beta \\
M_{1}, M_{2}, M_{3}, M_{4}: M_{1}=\omega_{1}-\omega_{2}, M_{2}=s+\omega_{2}, M_{3}=s+\omega_{1}, M_{4}=s+\gamma \\
E_{\omega 1 x}, E_{\omega 2 x}, E_{\gamma y}: E_{\omega 1 x}=\exp \left[-\left(s+\omega_{1}\right) x\right], E_{\omega 2 x} \\
=\exp \left[-\left(s+\omega_{2}\right) x\right], \\
E_{\gamma y}=\exp [-(s+\gamma) y]
\end{gathered}
$$

The possible states of channels and the transitions among them are shown in Figure 1.

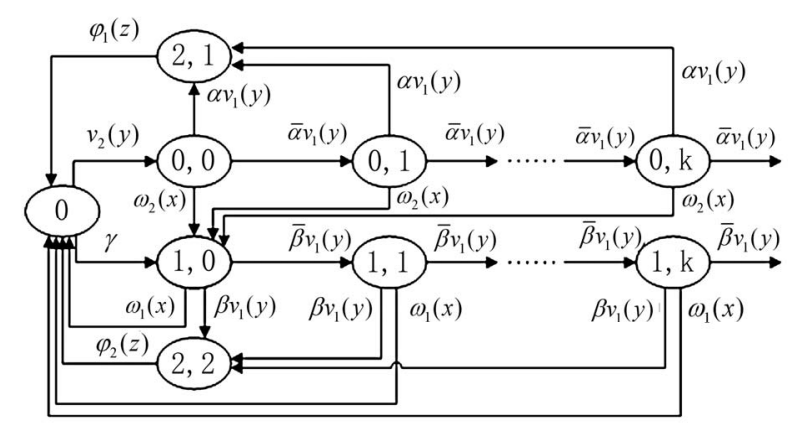

Figure 1. The state transition model of the generalized Markov process. 
According to Figure 1, a few new performance measures could be defined. The probability of the channel in available idle state, normal busy state and delay idle state are Available Idle Probability, Normal Busy Probability and Delay Idle Probability, respectively. Sensing Frequency is defined the frequency of the occurrence of channel sensing when the channel is in state $(i, k), i=0,1$. False alarm occurs if and only if the channel transfers from state $(0, k)(k=0,1,2 \cdots)$ to state $(2,1)$; while miss detection occurs if and only if the channel transfers from state $(1, k)(k=0,1,2 \cdots)$ to state $(2,2)$. So False Alarm Frequency and Miss Detection Frequency are defined accordingly. Also define $R_{1}(t), R_{2}(t), L_{0}(t) 9, L_{1}(t), L_{2}(t)$ and $L_{3}(t)$ are the Instantaneous Probability of Available Idle, Normal Busy, Delay Idle, Instantaneous Frequency of Sensing, False Alarm and Miss Detection at an arbitrary time $t$, respectively. Then define $R_{1}, R_{2}, L_{0}, L_{1}, L_{2}$ and $L_{3}$ are the steady state forms of $R_{1}(t), R_{2}(t)$, $L_{0}(t), L_{1}(t), L_{2}(t)$ and $L_{3}(t)$, respectively, e.g., $R_{1}=\lim _{t \rightarrow \infty} R_{1}(t)$.

In CR networks, each channel will go through one or all kinds of states in which available idle and normal busy generate rewards by the using for the transmission of CRs and PUs, respectively, delay idle and false alarm waste opportunities, miss detection induces interference to PUs, and sensing has overhead. It is assumed that the expected rewards per unit time generated by the channel are $e_{1}$ or $e_{2}$ when the channel is in available idle or normal busy, respectively. And the expected losses per unit time induced by the delay of channel is $c_{0}$, the expected cost of each sensing is $c_{1}$, the expected false alarm and miss detection expenses every time are $c_{2}$ and $c_{3}$, respectively. We also assumed that expected total rewards generated by the channel during $(0, \mathrm{t}]$ are $R$, then

$$
R(t)=\sum_{i=1}^{2} e_{i} \int_{0}^{t} R_{i}(t) d t-c_{0} \int_{0}^{t} L_{0}(t) d t-\sum_{j=1}^{3} c_{j} \int_{0}^{t} L_{j}(t) d t
$$

where $e_{1}, e_{2}, c_{0}, c_{1}, c_{2}$ and $c_{3}$ are weighting factors ( $e_{1}$ $\left.+e_{2}+c_{0}+c_{1}+c_{2}+c_{3}=1\right)$. Taking Laplace transform on both sides of (1), we have

$$
R^{*}(s)=\left[\sum_{i=1}^{2} e_{i} R_{i}^{*}(s)-c_{0} L_{0}^{*}(s)-\sum_{j=1}^{3} c_{j} L_{j}^{*}(s)\right] / s
$$

And the expected rewards per unit time in the steady state is

$$
\begin{aligned}
& R=\lim _{t \rightarrow \infty} R(t) / t=\lim _{s \rightarrow 0} s^{2} R^{*}(s) \\
& =\sum_{i=1}^{2} e_{i} R_{i}-c_{0} L_{0}-\sum_{j=1}^{3} c_{j} L_{j}
\end{aligned}
$$

where performance measures $R_{i}(i=1,2), L_{0}$ and $L_{j}(j=1$, $2,3)$ could be expressed as functions of the mean sensing period $E(T)$ (denoted by $\bar{x}$ ) and all of them will be illustrated how to obtain in Section 4. Then taking suitable value for $\bar{x}$ to make $\boldsymbol{R}$ maximum can bring out the optimal sensing period $\bar{x}^{\prime}$. That is

$$
\bar{x}^{\prime}=\underset{\bar{x}}{\arg \max }\left(\sum_{i=1}^{2} e_{i} R_{i}-c_{0} L_{0}-\sum_{j=1}^{3} c_{j} L_{j}\right)
$$

\section{Derivation of Performance Measures}

In order to derive performance measures for searching the optimal sensing period, the steady state probabilities with exact solutions in closed form should be calculated. How to calculate them using probability analysis and supplementary variables method is discussed below.

According to Figure 1, we have the following differential difference equations

$$
\begin{gathered}
{\left[\frac{\partial}{\partial t}+\frac{\partial}{\partial x}+\omega_{2}(x)+v_{1}(y)\right] p_{0 k}(t, x, y)=0 \quad k=0,1,2 \cdots(5)} \\
{\left[\frac{\partial}{\partial t}+\frac{\partial}{\partial x}+\omega_{1}(x)+v_{1}(y)\right] p_{10}(t, x, y)=\sum_{k=0}^{\infty} \mu p_{0 k}(t, x, y)(6)} \\
{\left[\frac{\partial}{\partial t}+\frac{\partial}{\partial x}+\omega_{1}(x)+v_{1}(y)\right] p_{1 k}(t, x, y)=0 \quad k=1,2 \cdots} \\
{\left[\frac{\partial}{\partial t}+\frac{\partial}{\partial y}+\gamma+v_{2}(y)\right] p_{0}(t, y)=0} \\
{\left[\frac{\partial}{\partial t}+\frac{\partial}{\partial z}+\varphi_{1}(z)\right] p_{21}(t, z)=0} \\
{\left[\frac{\partial}{\partial t}+\frac{\partial}{\partial z}+\varphi_{2}(z)\right] p_{22}(t, z)=0}
\end{gathered}
$$

The above equations are to be solved under the boundary conditions

$$
\begin{gathered}
p_{0}(t, 0) \\
=\sum_{k=0}^{\infty} \int_{0}^{\infty} \lambda p_{1 k}(t, x, y) d x d y+\int_{0}^{\infty} \varphi_{1}(z) p_{21}(t, z) d z \\
\quad+\int_{0}^{\infty} \varphi_{2}(z) p_{22}(t, z) d z+\delta(t) \\
p_{00}(t, 0)=\int_{0}^{\infty} v_{2}(y) p_{0}(t, y) d y \\
p_{0 k}(t, x, 0) \\
=\int_{0}^{\infty} \bar{\alpha} \omega_{2}(x) p_{0 k-1}(t, x, y) d x d y \quad k=1,2 \cdots \\
p_{10}(t, 0)=\int_{0}^{\infty} \gamma p_{0}(t, y) d y \\
p_{1 k}(t, x, 0)=\int_{0}^{\infty} \bar{\beta} v_{1}(y) p_{1 k-1}(t, x, y) d x d y \quad k=1,2 \cdots \\
p_{21}(t, 0)=\sum_{k=0}^{\infty} \int_{0}^{\infty} \alpha v_{1}(y) p_{0 k}(t, x, y) d x \\
p_{22}(t, 0)=\sum_{k=0}^{\infty} \int_{0}^{\infty} \beta v_{1}(y) p_{1 k}(t, x, y) d x d y
\end{gathered}
$$

And the initial conditions 


$$
p_{0}(0, y)=\delta(y)
$$

Taking Laplace transforms of Equations (5)-(18) with respect to $t$ and solving the equations, we can derive

$$
\begin{aligned}
& P_{0 k}^{*}(s, x, y) \\
& =M_{1} M_{4}\left(1-\bar{\alpha} f_{\omega 2}\right)\left(1-\bar{\beta} f_{\omega 1}\right) \bar{\alpha}^{k}\left(f_{\omega 2}\right)^{k} g_{\gamma} E_{\omega 2 x} \overline{F_{2}}(x) \text {. } \\
& \times \overline{G_{1}}(y) / D(s) \quad k=0,1,2 \ldots
\end{aligned}
$$

$$
\begin{aligned}
& P_{10}^{*}(s, x, y)= \\
& \left\{\left[\gamma M_{1}\left(1-g_{\gamma}\right)\left(1-\bar{\alpha} f_{\omega 2}\right)\left(1-\bar{\beta} f_{\omega 1}\right)-\mu M_{4} g_{\gamma}\left(1-\bar{\beta} f_{\omega 1}\right)\right] \times E_{\omega 1 x} \bar{F}_{1}(x) \bar{G}_{1}(y)+\mu M_{4} g_{\gamma}\left(1-\bar{\beta} f_{\omega 1}\right) E_{\omega 2 x} \bar{F}_{1}(x) \bar{G}_{1}(y)\right\} / D(s) \\
& P_{1 k}^{*}(s, x, y)= \\
& {\left[\bar{\beta} \gamma f_{\omega 1}\left(1-g_{\gamma}\right) M_{1}\left(1-\bar{\alpha} f_{\omega 2}\right)\left(1-\bar{\beta} f_{\omega 1}\right)+\omega_{2} \bar{\beta} g_{\gamma}\left(f_{\omega 2}-f_{\omega 1}\right) \times M_{4}\left(1-\bar{\beta} f_{\omega 1}\right)\right]\left(\bar{\beta} f_{\omega 1}\right)^{k-1} E_{\omega 1 x} \bar{F}_{1}(x) \bar{G}_{1}(y) / D(s) \quad k=1,2 \ldots} \\
& P_{0}^{*}(s, y)=\left(M_{4} M_{1}-M_{4} M_{1} \bar{\alpha} f_{\omega 2}-M_{4} M_{1} \bar{\beta} f_{\omega 1}+M_{4} M_{1} \bar{\alpha} \bar{\beta} \times f_{\omega 2} f_{\omega 1}\right) E_{\gamma y} \bar{G}_{2}(y) / D(s) \\
& P_{21}^{*}(s, z)=\left[\beta \gamma M_{1} f_{\omega 1}-\left(\beta \gamma M_{1}+\beta \omega_{2} M_{4}\right) f_{\omega 1} g_{\gamma}-\beta \gamma M_{1} \bar{\alpha} f_{\omega 2} f_{\omega 1}+\beta \omega_{2} M_{4} f_{\omega 2} g_{\gamma}+\beta \gamma M_{1} \bar{\alpha} f_{\omega 2} f_{\omega 1} g_{\gamma}\right] \exp (-s z) \overline{H_{1}}(z) / D(s) \\
& P_{22}^{*}(s, z)=\left(\alpha M_{1} M_{4} f_{\omega 2} g_{\gamma}-\alpha M_{1} M_{4} \bar{\beta} f_{\omega 2} f_{\omega 1} g_{\gamma}\right) \exp (-s z) \times \overline{H_{2}}(z) / D(s)
\end{aligned}
$$

where

$$
\begin{aligned}
& D(s)=\left(M_{1} M_{4}-M_{1} \omega_{1} \gamma / M_{3}\right)+\left(M_{1} \omega_{1} \gamma / M_{3}-M_{1} M_{4} \bar{\beta} f_{\omega 1}\right)+\left(M_{1} \bar{\alpha} \times \omega_{1} \gamma / M_{3}-M_{1} \bar{\alpha} M_{4}\right) f_{\omega 2}+\left(M_{1} \bar{\alpha} \bar{\beta} M_{4}-M_{1} \bar{\alpha} \omega_{1} \gamma / M_{4}\right) f_{\omega 2} f_{\omega 1} \\
& -\left(M_{1} \beta \gamma \times f_{\omega 1}-M_{1} \beta \gamma \bar{\alpha} f_{\omega 1} f_{\omega 2}\right) h_{1}^{*}(s)-\left\{\left[M_{4} \omega_{1} \omega_{2} / M_{2}-\left(M_{4} \omega_{1} \omega_{2}+M_{1} \omega_{1} \gamma\right) / M_{3}\right]+\left[\left(M_{3} \omega_{1} \omega_{2}+M_{1} \omega_{1} \gamma\right) / M_{3}-\left(M_{4} \omega_{1}-\mu \bar{\beta}\right) / M_{2}\right] f_{\omega 1}\right. \\
& \left.+\left[\left(M_{4} \omega_{1} \omega_{2} \bar{\beta}+M_{1} \times \omega_{1} \gamma \bar{\alpha}\right) / M_{3}-M_{4} \omega_{1} \omega_{2} / M_{2}\right] f_{\omega 2}+\left[M_{4} \omega_{1} \omega_{2} \bar{\beta} / M_{2}-\left(M_{4} \omega_{1} \omega_{2} \bar{\beta}+\gamma \bar{\alpha} M_{1}\right) / M_{3}\right] f_{\omega 2} f_{\omega 1}\right\} g_{\gamma} \\
& -\left[M_{4} \beta \omega_{2} f_{\omega 2}-\left(s \beta \omega_{2}-\omega_{1} \beta \gamma\right) f_{\omega 1}+M_{1} \beta \gamma \bar{\alpha} f_{\omega 2} f_{\omega 1}\right] h_{1}^{*}(s) \times g_{\gamma}-\left(M_{1} M_{4} \alpha f_{\omega 2}-M_{1} M_{4} \alpha \bar{\beta} f_{\omega 2} f_{\omega 1}\right) h_{2}^{*}(s) g_{\gamma}
\end{aligned}
$$

In accordance with the definitions of $R_{1}(t)$ and $R_{2}(t)$, we obtain

$$
\begin{aligned}
& R_{1}(t)=\sum_{k=0}^{\infty} \int_{0}^{\infty} P_{0 k}(t, x) d x \\
& R_{2}(t)=\sum_{k=0}^{\infty} \int_{0}^{\infty} P_{1 k}(t, x) d x
\end{aligned}
$$

Taking Laplace transform on both sides of Equations (26), (27) and using Equations (19)-(25), we get

$$
\begin{aligned}
& R_{1}^{*}(s) \\
&=\left(M_{1} M_{4} g_{\gamma}-M_{1} M_{4} \bar{\beta} f_{\omega 1} g_{\gamma}\right) \overline{F_{2}^{*}}\left(s+\omega_{2}\right) / D(s) s \\
& R_{2}^{*}(s)=\left[\gamma M_{1}-\left(\gamma M_{1}+\omega_{2} M_{4}\right) g_{\gamma}-\gamma M_{1} \bar{\alpha} f_{\omega_{2}}\right. \\
&\left.+\left(\gamma M_{1} \bar{\alpha}+\omega_{2} \bar{\beta} M_{4}\right) g_{\gamma} f_{\omega_{2}}\right] \overline{F_{1}^{*}}\left(s+\omega_{1}\right) \\
&+\left(\omega_{2} M_{4} g_{\gamma}-\omega_{2} M_{4} \times \bar{\beta} f_{\omega 1} g_{\gamma}\right) \overline{F_{2}^{*}}\left(s+\omega_{2}\right)
\end{aligned}
$$

where, $\mathrm{D}(\mathrm{s})$ is given by Equation (25). By applying the limiting theorem of Laplace transform and L'Hospital's rule, we get

$$
\begin{aligned}
R_{1} & =\lim _{t \rightarrow \infty} R_{1}(t)=\lim _{s \rightarrow 0} s R_{1}^{*}(s) \\
& =\left(M_{1} \gamma \hat{g}_{\gamma}-M_{1} \gamma \bar{\beta} \hat{f}_{\omega 1} \hat{g}_{\gamma}\right) \overline{F_{2}^{*}}\left(\omega_{2}\right) / D
\end{aligned}
$$

$$
\begin{aligned}
R_{2}=\lim _{t \rightarrow \infty} R_{2}(t)=\lim _{s \rightarrow 0} s R_{2}^{*}(s) \\
=\left[\gamma M_{1}-\gamma \omega_{1} \hat{g}_{\gamma}-\gamma M_{1} \bar{\alpha} \hat{f}_{\omega 2}+\left(\gamma M_{1} \bar{\alpha}+\gamma \omega_{2} \bar{\beta}\right) \hat{g}_{\gamma} \hat{f}_{\omega 2}\right] \\
\quad \times \overline{F_{1}^{*}}\left(\omega_{1}\right)+\left[\left(\omega_{2} \gamma \hat{g}_{\gamma}-\omega_{2} \gamma \bar{\beta} \hat{g}_{\gamma} \hat{f}_{\omega 1}\right) \overline{F_{2}^{*}}\left(\omega_{2}\right)\right] / D
\end{aligned}
$$

where

$$
\begin{aligned}
& D=D^{\prime}(0)=M_{1}\left(\omega_{1}+\gamma\right) / \omega_{1}-\left[M_{1}\left(\gamma+\bar{\beta} \omega_{1}\right) / \omega_{1}\right] \hat{f}_{\omega_{1}} \\
& -\left[M_{1} \bar{\alpha}\left(\omega_{1}+\gamma\right) / \omega_{1}\right] \hat{f}_{\omega_{2}}+\left[M_{1} \bar{\alpha}\left(\gamma+\bar{\beta} \omega_{1}\right) / \omega_{1}\right] \hat{f}_{\omega_{2}} \hat{f}_{\omega_{1}} \\
& -\left\{M_{1}\left(\omega_{2}-\gamma\right) / \omega_{2}+\left\{\left[\bar{\beta}\left(\omega_{2}{ }^{2}-\omega_{1} \omega_{2}+\gamma \omega_{1}\right)-\omega_{2} \gamma\right] / \omega_{2}\right\} \hat{f}_{\omega_{1}}\right. \\
& +\left\{\left[\gamma \omega_{2}^{2}(\beta-\alpha)-\omega_{1} \gamma \omega_{2} \bar{\alpha}-\omega_{1} \omega_{2} M_{1} \bar{\alpha}+\gamma \omega_{1}^{2}\right] / \omega_{1} \omega_{2}\right\} \times \hat{f}_{\omega_{2}} \\
& \left.+\left\{\left[\omega_{1} \omega_{2} \bar{\alpha} \bar{\beta} M_{1}+\gamma \bar{\beta}\left(\omega_{2}{ }^{2}-\omega_{1}^{2}\right)+\gamma \omega_{2} \bar{\alpha} M_{1}\right] / \omega_{1} \omega_{2}\right\} \hat{f}_{\omega_{2}} \hat{f}_{\omega_{1}}\right\} \hat{g}_{\gamma} \\
& +\left(\beta \gamma \times M_{1} \hat{f}_{\omega_{1}}-\beta \gamma M_{1} \bar{\alpha} \hat{f}_{\omega_{2}} \hat{f}_{\omega_{1}}\right)\left(1 / \varphi_{1}\right)+\left(\beta \gamma \omega_{2} \hat{f}_{\omega_{2}}-\beta \gamma \omega_{1} \hat{f}_{\omega_{1}}\right. \\
& \left.+\beta \gamma M_{1} \bar{\alpha} \hat{f}_{\omega_{2}} \hat{f}_{\omega_{1}}\right) \times \hat{g}_{\gamma}\left(1 / \varphi_{1}\right) \\
& +\left(\gamma \alpha M_{1} \hat{f}_{\omega_{2}}-\gamma \alpha M_{1} \bar{\beta} \hat{f}_{\omega_{2}} \hat{f}_{\omega_{1}}\right) \hat{g}_{\gamma}\left(1 / \varphi_{2}\right)
\end{aligned}
$$

With the same derivation of $R_{1}$ and $R_{2}$, we can get

$$
\begin{aligned}
& L_{0}=\left(\gamma M_{1}-\gamma M_{1} \bar{\alpha} \hat{f}_{\omega_{2}}-\gamma M_{1} \bar{\beta} \hat{f}_{\omega_{1}}\right. \\
& \left.S+\gamma M_{1} \bar{\alpha} \bar{\beta} \hat{f}_{\omega_{2}} \hat{f}_{\omega_{1}}\right) \overline{G_{2}{ }^{*}}(\gamma) / D
\end{aligned}
$$

According to Figure 1, as the channel is in state $(i, k), i=0,1$, it is being sensed. Using state transfer frequency formula shown in [9], we get

$$
L_{1}(t)=\sum_{k=0}^{\infty} \int_{0}^{\infty} \omega(x)\left[P_{0 k}(t, x)+P_{1 k}(t, x)\right] d x
$$

Taking Laplace transform on Equation (34) and using Equations (19)-(25), we obtain

$$
\begin{aligned}
L_{1}^{*}(s) & =\left\{\left(M_{1} M_{4} g_{\gamma}-M_{1} M_{4} \bar{\beta} f_{\omega_{1}} g_{\gamma}\right) f_{\omega_{2}}\right. \\
& +\left\{\gamma M_{1}-\left(\gamma M_{1}+\omega_{2} M_{4}\right)\right] g_{\gamma}-\gamma M_{1} \bar{\alpha} f_{\omega_{2}} \\
& \left.+\left(\gamma M_{1} \bar{\alpha}+\omega_{2} M_{4} \bar{\beta}\right) g_{\gamma} f_{\omega_{2}}\right\} \times f_{\omega_{1}} \\
& \left.+\left(\omega_{2} M_{4} g_{\gamma}-\omega_{2} M_{4} \bar{\beta} g_{\gamma} f_{\omega_{1}}\right) f_{\omega_{2}}\right\} / D(s)
\end{aligned}
$$

Then 


$$
\begin{aligned}
L_{1}=\lim _{t \rightarrow \infty} L_{1}(t)=\lim _{s \rightarrow 0} s L_{1}^{*}(s) \\
=\left[\gamma M_{1} \hat{f}_{\omega 1}+\omega_{2} \gamma \hat{f}_{\omega_{2}} \hat{g}_{\gamma}-\gamma \omega_{1} \hat{f}_{\omega 1} \hat{g}_{\gamma}-\gamma M_{1} \bar{\alpha} \hat{f}_{\omega_{2}} \hat{f}_{\omega 1}\right. \\
\left.\quad-\gamma M_{1}(\alpha-\beta) \hat{f}_{\omega_{2}} \hat{f}_{\omega 1} \hat{g}_{\gamma}\right] / D \\
\text { Similarly, we can derive } \\
L_{2}=\left(\beta \gamma M_{1} \hat{f}_{\omega 1}-\beta \gamma \omega_{1} \hat{f}_{\omega 1} \hat{g}_{\gamma}-\beta \gamma M_{1} \bar{\alpha} \hat{f}_{\omega 2} \hat{f}_{\omega 1}\right. \\
\left.\quad+\beta \omega_{2} \gamma \hat{f}_{\omega_{2}} \hat{g}_{\gamma}+\beta \gamma M_{1} \bar{\alpha} \hat{f}_{\omega_{2}} \hat{f}_{\omega 1} \hat{g}_{\gamma}\right) / D \\
L_{3}=\left(\alpha \gamma M_{1} \hat{f}_{\omega_{2}} \hat{g}_{\gamma}-\alpha \gamma M_{1} \bar{\beta} \hat{f}_{\omega_{2}} \hat{f}_{\omega 1} \hat{g}_{\gamma}\right) / D
\end{aligned}
$$

Substituting $R_{i}(i=1,2), L_{0}$ and $L_{j}(j=1,2,3)$ by (30), (31), (33), (36), (37) and (38) into (4), respectively, the optimal sensing period $\vec{x}^{\prime}$ is determined by $F_{i}(t)$, i.e., the distributions of sojourn time of normal busy and available idle. For the sojourn time of normal busy is used for transmission of PUs themselves and that of available idle can be utilized by CRs' transmission when PUs have no data to transmit, $F_{i}(t)$ are usually determined by the traffic generated by PUs services in practical networks.

\section{The Applications of Sensing Period Optimization}

This section illustrates the applications of the analytical model developed here by using examples involving PU systems with both voice and data traffic.

\subsection{Optimization for PUs with Voice Traffic}

A typical phone conversation is marked by periods of active talking/talk spurts (or ON periods) interleaved by silence/ listening periods (or OFF periods). The duration of each period is exponentially distributed, i.e., the sojourn time of normal busy and available idle follow exponential distributions with probability density functions $f_{i}(t)=\lambda_{i} e^{-\lambda_{i} t}$, and means $\lambda_{i}^{-1}$ are constants.

It is a special case for analytical model mentioned above in which $\omega_{i}^{-1} s$ can be substitute by $\lambda_{i}^{-1} z$. The transient rate of available idle to normal busy and that of normal busy to delay idle are thus reduced to constants $\lambda_{i}$.

\subsection{Optimization for PUs with Data Traffic}

In the past, exponential distributions are also frequently employed to model interarrival times of data calls for its simplicity, but exponential distributions may not be appropriate in modeling data traffic. Taking Email, an important application that constitutes a high percentage of internet traffic, as an example, its traffic can also be characterized by ON/OFF states. During the ON-state an email could be transmitted or received, and during the OFF-state a client is writing or reading an email. According to traffic models included in the UMTS Forum 3G traffic and ITU RM.2072, the Pareto distribution, which is one of popular heavy-tailed distributions, can be used to close capture the nature of Email traffic for both $\mathrm{ON}$ and OFF state, i.e., the sojourn time of normal busy and available idle follow Pareto distributions with probability density functions $f_{i}(t)=\frac{\alpha_{i} \beta_{i}^{\alpha_{i}}}{t^{\alpha_{i}+1}}, t>\alpha_{i}$, and means $\alpha_{i} \beta_{i} /\left(\alpha_{i}-1\right)$, where $\alpha_{i}>0$ is called the shape parameter and $\beta_{i}>0$ is called the scale parameter.

In order to derive performance measures to search the optimal sensing period, $\frac{\alpha_{i} \beta_{i}^{\alpha_{i}}}{t^{\alpha_{i}+1}}$ and means $\alpha_{i} \beta_{i} /\left(\alpha_{i}-1\right)$ should substitute $f_{i}(t)$ and $\omega_{i}^{-1}$ in Equations (5)-(18).

\section{Numerical Results and Discussions}

Through numerical experiments, we examine the impact of sensing period selection on maximum expected rewards of the channel for different traffic types under various channel parameters in this section.

\subsection{Performance Analysis}

According to the characteristics of channels, we first set the channel parameters as the following $\gamma=0.02$, $\varphi_{1}=0.033, \varphi_{2}=0.1, \alpha=0.2, \beta=0.1, e_{1}=e_{2}=0.1, c_{0}=c_{2}=0.05$, $c_{1}=0.2, \quad c_{3}=0.5$, and let us assume that $T_{0}$ follows the uniform distribution with parameters $\bar{y}-10$ and $\bar{y}+10$, written $\mathrm{T}_{0} \sim U(\bar{y}-10, \bar{y}+10)$, and $\bar{y}=10$.

Two traffic types of PUs are considered, i.e., Type I: voice traffic and Type II: data traffic. In the case of voice traffic, the sojourn time of normal busy and available idle follow exponential distributions with $\lambda_{1}=0.04$, $\lambda_{2}=0.01$; for data traffic, the sojourn time of normal busy and available idle follow Pareto distributions with $\alpha_{1}=0.8, \beta_{1}=5$ and $\alpha_{2}=2.5, \beta_{2}=60$, i.e., means are $\omega_{1}=0.04$ and $\omega_{2}=0.01$, respectively. Here $\omega_{1}=\lambda_{1}$ and $\omega_{2}=\lambda_{2}$ are selected for easy comparison.

To validate the feasibility of the proposed analytical model for sensing period optimization, numerical examples are carried out for the following three sensing schemes, i.e.,

1) Scheme 1: $T=\bar{x}$, that is, it is exactly a fixed period sensing scheme that sensing is performed at once where $T=\bar{x}$.

2) Scheme 2: $T$ follows the uniform distribution with parameters $\bar{x}-1$ and $\bar{x}+1$, written $T \sim U=(\bar{x}-1, \bar{x}+1)$.

3) Scheme 3: $T$ follows the exponential distribution with parameter $\bar{x}$, written $T \sim E P(\bar{x})$. 
With MATLAB, from (4) we can obtain the optimal sensing periods $\vec{x}$ and the maximum expected rewards $\boldsymbol{R}$ of the channel per unit time in the steady state for each sensing scheme. Figure 2 and Figure 3 illustrate the expected rewards of the channel for various values of $\bar{x}$ under Type I and Type II traffic types respectively.

For Type I voice traffic, Figure 2 shows that the maximum expected rewards of the channel is varied according to the distribution of sensing period $T$. The optimal sensing periods for each scheme are $\vec{x}^{\prime}=\{6.5$, $13.2,7.8\}$ and the maximum expected rewards are $R=\{109.8,106.1,114.9\}$. So the optimal sensing scheme is sensing randomly with sensing period $T$ following the exponential distribution when $\bar{x}=7.8$ under the above-mentioned channel parameters, for the scheme obtained $R=114.9$ is maximum compared to other two schemes.

For Type II data traffic, Figure 3 shows that the optimal sensing periods for each scheme are $\vec{x}=\{10.7,17.9,11.3\}$ and the maximum expected rewards are $R=\{77.7,113.7,90.2\}$. From Figure 3, it can easily find that the optimal sensing scheme is sensing randomly with sensing period $T$ following the uniform distribution. One point that deserves mention is that, compared to the voice traffic, there are significant differences in maximum expected rewards among the three schemes in the case of data traffic.

\subsection{Analysis of Channel Parameters}

In practical, the optimal sensing scheme is different with different sets of channel parameters. Next, we study effects of the setting of various channel parameters on the optimal sensing scheme.

1) Effects of Distribution Parameters: in both voice

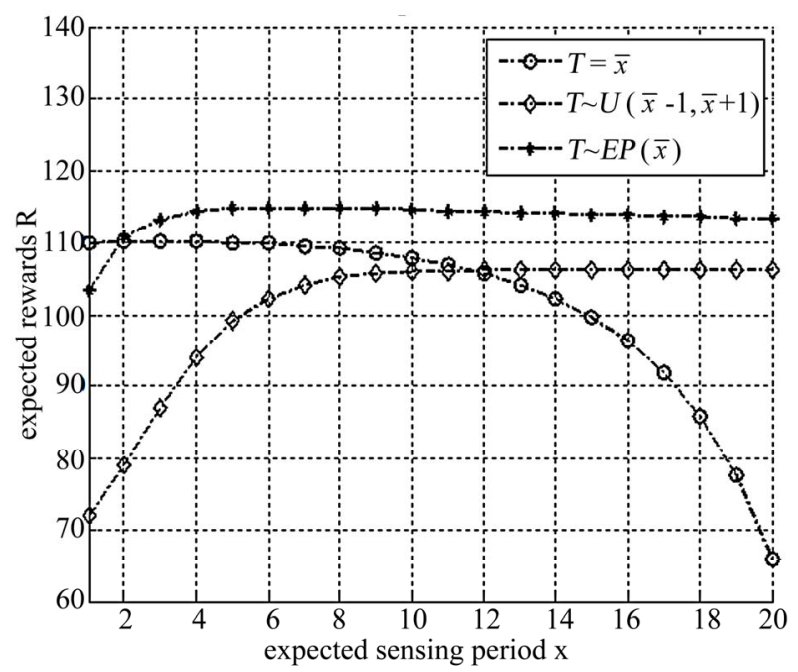

Figure 2. expected rewards vs. expected sensing period for voice traffic.

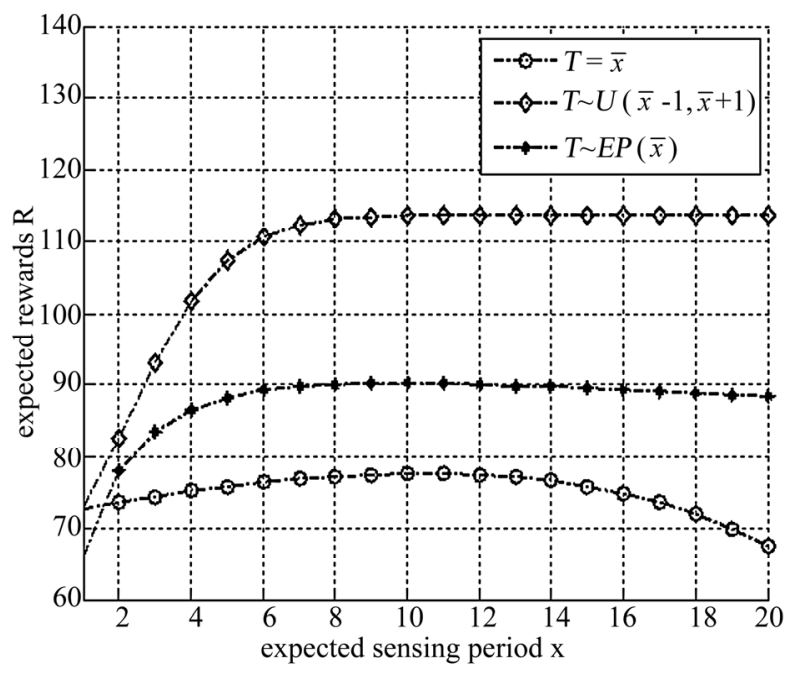

Figure 3. expected rewards vs. expected sensing period for data traffic.

Table 1. The Optimal Rewards $R$ Vs. Optimal Mean Sensing Period $\vec{x}$ for Various Distribution Parameters under $e_{1}=e_{2}=0.1, \quad c_{0}=c_{2}=0.05, c_{1}=0.2, c_{3}=0.5, \alpha=0.2, \beta=0.1$, $\mathrm{T}_{0} \sim U(\bar{y}-10, \bar{y}+10)$ and $\bar{y}=10$.

\begin{tabular}{lcllll}
\hline$\left\{\omega_{1} / \lambda_{1}, \omega_{2} / \lambda_{2}\right.$ & & \multicolumn{2}{c}{ Type I } & \multicolumn{2}{c}{ Type II } \\
$\left.\gamma, \varphi_{1}, \varphi_{2}\right\}$ & $T$ & $\bar{x}^{\prime}$ & $R$ & $\bar{x}^{\prime}$ & $R$ \\
\hline$(0.01,0.04$ & $T=\bar{x}$ & 7.8 & 108.1 & 6.5 & 121.3 \\
$, 0.02,0.03$ & $T \sim U=(\bar{x}-1, \bar{x}+1)$ & 10.2 & 106.3 & 9.2 & 86.5 \\
$3,0.1)$ & $T \sim E P(\bar{x})$ & 11.8 & 105.5 & 7.4 & 94.6 \\
\hline$(0.04,0.01$ & $T=\bar{x}$ & 9.4 & 110.5 & 10.7 & 79.8 \\
$, 0.05,0.03$ & $T \sim U=(\bar{x}-1, \bar{x}+1)$ & 11.9 & 109.8 & 8.8 & 99.6 \\
$3,0.1)$ & $T \sim E P(\bar{x})$ & 10.2 & 112.3 & 5.9 & 128.9 \\
\hline \multirow{2}{*}{$(0.04,0.01$} & $T=\bar{x}$ & 10.5 & 97.5 & 15.1 & 108.2 \\
$, 0.02,0.1,0$ & $T \sim U=(\bar{x}-1, \bar{x}+1)$ & 9.3 & 99.3 & 17.5 & 132.4 \\
$.033)$ & $T \sim E P(\bar{x})$ & 8.9 & 101.6 & 3.9 & 86.7 \\
\hline
\end{tabular}

traffic and data traffic, the distribution parameters $\lambda_{1}, \lambda_{2}, \gamma, \varphi_{1}, \varphi_{2}$ and $\omega_{1}, \omega_{2}, \gamma, \varphi_{1}, \varphi_{2}$ directly reflect the sojourn time of normal busy, available idle, delay idle, false alarm and miss detection state, respectively. The distribution parameters $\omega_{1} / \lambda_{1}=0.04, \omega_{2} / \lambda_{2}=0.01$ $\left(\gamma=0.02, \varphi_{1}=0.033, \varphi_{2}=0.1\right)$ show that the sojourn time of available idle is longest, which means low or moderate PU traffics are chosen. Other three distribution parameters are chosen for 3 sensing schemes, and the optimal rewards $R$ vs. the optimal mean sensing period $\vec{x}$ are shown in Table 1, respectively. a) $\left(\omega_{1} / \lambda_{1}, \omega_{2} / \lambda_{2}, \gamma, \varphi_{1}, \varphi_{2}\right)$ is $(0.01,0.04,0.02,0.033,0.1)$. The sojourn time of normal busy is relatively long and less opportunities can be used by transmission of CRs; $b)\left(\omega_{1} / \lambda_{1}, \omega_{2} / \lambda_{2}, \gamma, \varphi_{1}, \varphi_{2}\right)$ is $(0.04,0.01,0.05,0.033,0.1)$. The sojourn time of delay idle is reduced so that more opportunities can be used by CRs than 1$)$; and c) $\left(\omega_{1} / \lambda_{1}, \omega_{2} / \lambda_{2}, \gamma, \varphi_{1}, \varphi_{2}\right)$ is $(0.04$, $0.01,0.02,0.1,0.033)$. The sojourn time of miss detec- 
tion is longer than that of false alarm. From Table 1, we clearly see that the optimal sensing scheme is different with regard to different distribution parameters.

2) Effects of Weighting Factors: Weighting factors $e_{1}$, $e_{2}, c_{0}, c_{1}, c_{2}$ and $c_{3}$ can be treated as indexes regarding the importance of $R_{1}, R_{2}, L_{0}, L_{1}$ and $L_{2}$. Generally speaking, the PU applications, such as GPS, can only endure minor interference for acceptable Quality of Service (QOS). For such applications, the cost of interference induced by miss detection is more important and $c_{3}$ is obviously bigger than all other factors as shown in above-chosen setting $e_{1}=e_{2}=0.1, c_{0}=c_{2}=0.05, c_{1}=0.2, \quad\left(c_{3}=0.5\right)$. Table 2 shows optimal rewards $R$ and the optimal mean sensing period $\vec{x}$ for two different network applications : a) In the case where the PU is not sensitive to interference, the weighting factors $c_{0}$ and $c_{2}$ are both larger than other factors in order to maximize the utilization of existing opportunities. Here $\left(e_{1}, e_{2}, c_{0}, c_{1}, c_{2}, c_{3}\right)$ is set to $(0.1,0.1,0.35,0.05,0.35,0.05)$. b) For energy-constrained CR networks such as sensors and mobile ad hoc applications, frequent sensing is undesirable for high energy overhead, so the weighting factor $c_{1}$ should be set relatively large and $(0.1,0.1,0.05,0.5,0.05,0.1)$ is chosen. This table demonstrates that the optimal sensing scheme is also different with regard to different weighting factors and from the Table 2 one can easily find which scheme performs best.

\subsection{Performance Comparison for Different Traffic Types}

According to the proposed analytical model for sensing period optimization, a sensing scheme with a bigger expected reward performs better. Figure 2, Figure 3, Table 1 and Table 2 show that the optimal sensing scheme is different for different traffic types. In Figure 4, we show the expected rewards for different schemes under $\gamma=0.02, \quad \varphi_{1}=0.033, \quad \varphi_{2}=0.1, \quad \beta=0.1, e_{1}=e_{2}=0.1$, $c_{0}=c_{2}=0.05, c_{1}=0.2, c_{3}=0.5$, and two different situations

Table 2. The optimal rewards $R$ vs. optimal mean sensing period $\vec{x}^{\prime}$ for various weighting factors under $\lambda=0.04$, $\mu=0.01, \quad \gamma=0.02, \varphi_{1}=0.033, \varphi_{2}=0.1, \quad \alpha=0.2, \beta=0.1$ $\mathrm{T}_{0} \sim U(\bar{y}-10, \bar{y}+10)$, and $\bar{y}=10$.

\begin{tabular}{cccccc}
\hline $\begin{array}{c}\left(e_{1}, e_{2},\right. \\
c_{0}, c_{1}, c_{2},\end{array}$ & $T$ & \multicolumn{2}{c}{ Type I } & \multicolumn{2}{c}{ Type II } \\
$\left.c_{3}\right)$ & & $\bar{x}^{\prime}$ & $R$ & $\bar{x}^{\prime}$ & $R$ \\
\hline$(0.1,0.1$, & $T=\bar{x}$ & 10.4 & 107.6 & 9.7 & 73.9 \\
$0.35,0.0$ & $T \sim U=(\bar{x}-1, \bar{x}+1)$ & 11.3 & 111.4 & 14.5 & 98.2 \\
$5,0.35,0$. & $T \sim E P(\bar{x})$ & 8.9 & 105.3 & 10.3 & 114.6 \\
$05)$ & $T=\bar{x}$ & 7.5 & 98.8 & 11.8 & 89.2 \\
$(0.1,0.1$, & $T \sim U=(\bar{x}-1, \bar{x}+1)$ & 15.2 & 95.6 & 6.2 & 125.5 \\
$0.05,0.5$, & $T \sim E P(\bar{x})$ & 12.6 & 101.2 & 4.9 & 75.4 \\
$0.05,0.1)$ & $T \sim$ & & & & \\
\hline
\end{tabular}

for voice traffic: 1) fixed $\lambda_{2}(=0.15)$ and various $\lambda_{1}$; and 2) fixed $\lambda_{1}(=0.2)$ and various $\lambda_{2}$, and two different situations for data traffic: 1) fixed $\omega_{2}(=0.15)$ and various $\omega_{1}$; and 2$)$ fixed $\omega_{1} \quad(=0.2)$ and various $\omega_{2}$.

As shown in Figure 4(a) and Figure 4(b), varying $\lambda_{1}$ $\left(\lambda_{2}\right)$ among 0.1 and 1 , we observed that the performance of voice traffic is not sensitive to which sensing scheme is preferred. However, for data traffic, with the variation of $\omega_{1}$ and $\omega_{2}$, the optimal sensing scheme is changing among three sensing schemes and the maximum expected rewards have big different, as shown in Figure 4(c) and Figure 4(d). The possible reason is that the optimal sensing period for voice traffic is depending only on a constant mean value of the transmission time for primary users and cognitive radios whereas performance of data traffic are affected by not only mean value but also the distributions of the time of transmission for primary users and cognitive radios.

From the above discussions, we suggest that: 1) for voice traffic, the maximum expected rewards of three sensing scheme is close to each other. In real system, a fixed period sensing scheme is preferred for simplicity; and 2) however, the situation changes dramatically for data traffic, the maximum expected rewards of three sensing scheme are far different. The analytical model proposed can be used to search the optimal sensing scheme, and the analysis can be easily extended to that of any other distribution of sensing period $T$.

\section{Conclusions and Future Work}

This paper dealt with the random sensing period scheme in CR networks. An efficient generalized Markov analytical model for sensing period optimization was proposed and studied. How our proposed analytical model can be applied to PU systems with both voice and data traffic was also discussed. Both numerical results and analysis for various channel parameters and traffic types of PUs were obtained and compared. We found that sensing period does affect the maximum expected rewards of the channel, and the proposed analytical model is valid for the analysis of the case where the sensing period, the transmission time for primary users and cognitive radios are all following arbitrary distributions.

In this work, we assume that the sensing period for each channel is different, i.e. the sensing period is asynchronous for all channels. The proposed scheme is suitable for the scenario where each CR only sense the channel for its operating. If each $\mathrm{CR}$ is responsible for sensing more than one channel, the intelligence schedule algorithm of sensing period should be used to negotiate among CRs because the quiet period of each channel is also asynchronous. In future, we would like to develop practical schedule mechanisms or protocols, which deal 




(a)

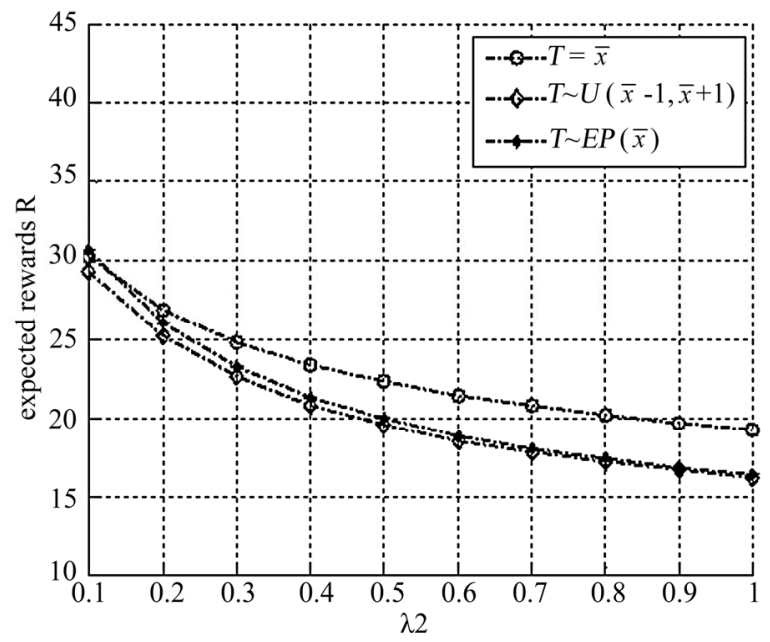

(c)

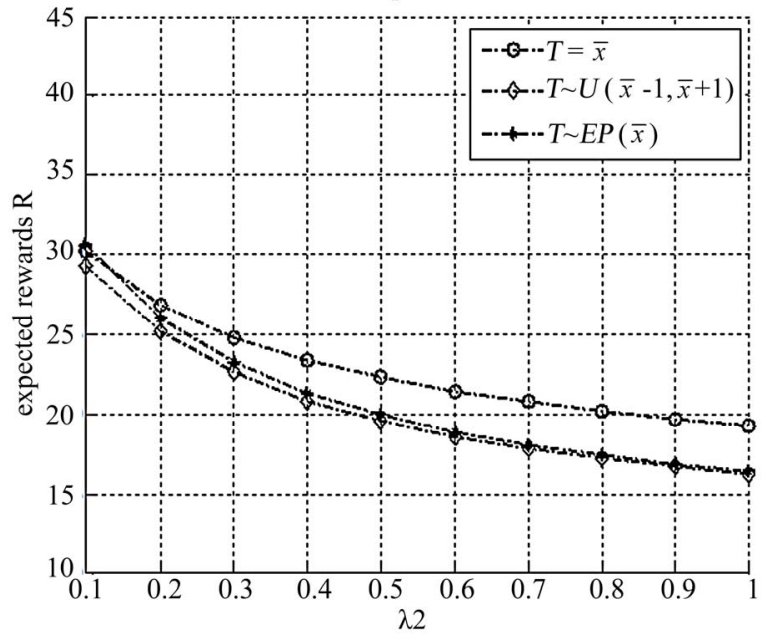

(b)

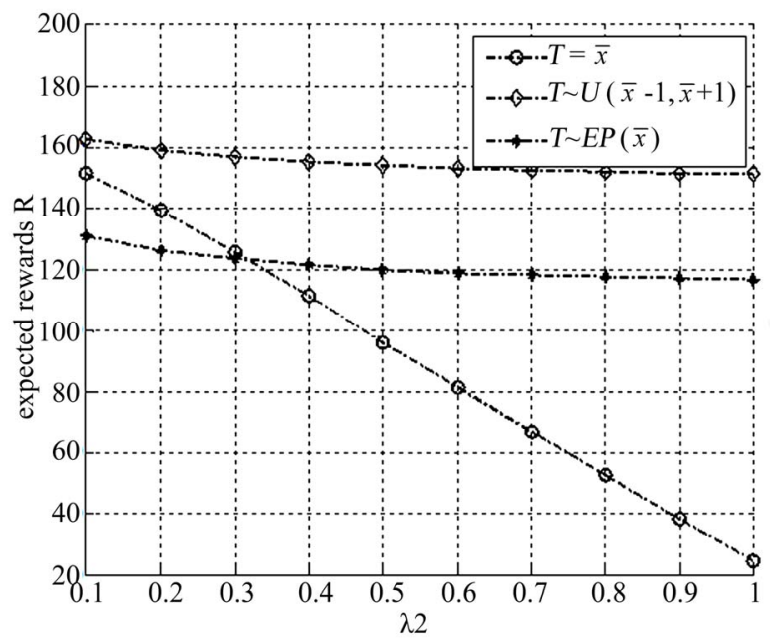

(d)

Figure 4. Performance comparison of different traffic types under $\gamma=0.02, \quad \varphi_{1}=0.033, \varphi_{2}=0.1, \quad \alpha=0.2, \quad \beta=0.1$, $e_{1}=e_{2}=0.1, c_{0}=c_{2}=0.05, c_{1}=0.2, c_{3}=0.5$ with (a) $\lambda_{2}=\mathbf{0 . 1 5}$, (b) $\lambda_{1}=\mathbf{0 . 2}$, (c) $\omega_{2}=\mathbf{0 . 1 5}$, and (d) $\omega_{1}=\mathbf{0 . 2}$.

with the coordination of channel sensing among CRs, to make the proposed sensing scheme more effective in real CR networks.

\section{Acknowledgements}

This work was supported by Natural Science Foundation of China under Grant No. 60772110.

\section{References}

[1] Y. C. Liang, W. S. Leon, Y. H. Zeng, et al., "System description and operation principles for IEEE 802.22 WRANs," IEEE 802.22-05/0094r4, http://www.ieee802. $\operatorname{org} / 22 /$.

[2] V. K. N. Lau, R. S. Cheng, R. D. Murch, et al., “Adap- tive quiet period control," IEEE 802.22-06/0082r0, http:// www.ieee802.org/22/.

[3] W. D. Hu, D. Willkomm, M. Abusubaih, et al., "Cognitive Radios for dynamic spectrum access-dynamic frequency hopping communities for efficient IEEE 802.22 operation," IEEE Communications Magazine, Vol. 45, No. 5, pp. 88-87, 2007.

[4] T. Shu, S. G. Cui, and M. Krunz, "Medium access control for Multi-Channel parallel transmission in Cognitive Radio networks," In proceedings of IEEE GLOBECOM, San Francisco, pp. 1-5, 2006.

[5] H. Kim and K. G. Shin, "Efficient discovery of spectrum opportunities with MAC-Layer sensing in Cognitive Radio networks," IEEE Transactions on Mobile Computing, Vol. 7, No. 5, pp. 533-545, 2008.

[6] Y. Zhang, C. Y. Feng, and C. L. Guo, "A flexible sensing period mechanism of spectrum sensing in cognitive radio 
Networks," The Journal of China Universities of Posts and Telecommunications, Vol. 31 No. 2, pp. 128-131, 2008.

[7] C. L. Guo, Z. M. Zeng, C. Y. Feng, and Z. Q. Liu, “An asynchronous spectrum sensing period optimization model and adaptive fuzzy adjustment algorithm," Journal of Electronics \& Information Technology, Vol. 31, No. 4, pp. $920-924,2009$.
[8] C. Han, J. Wang, and S. Li, "A spectrum exchange mechanism in cognitive radio contexts," In proceedings of IEEE PIMRC, Finland, pp. 1-5, 2006.

[9] D. H. Shi, "A new method for calculating the mean failure numbers of a repairable system during $(0, t)$," Acta Mathematicac Applicatae Sinica, Vol. 8, No. 1, pp. 101110, 1985. 